\title{
A CONVOLUTION EQUATION ON A COMPACT INTERVAL
}

\author{
P. E. GREENWOOD ${ }^{1}$
}

1. Introduction. We present two theorems which give sufficient conditions for the existence of a solution, $s$, of the convolution equation $g(x)=\int_{J} W(x-y) d s(y)$ (briefly, $g=W * s$ ). Here $J$ is, say, the compact real interval $[0,1], g$ is an $L^{2}(J, d x)$ function, and $W$ is a complex-valued function on $[-1,1]$ with the following property: The operator $\left[D^{2} W *\right]: f \rightarrow D^{2} W * f=\int_{-1}^{1} f(x-y) d D^{2} W(y)$ maps $L^{2}(J, d x)$ into itself, where differentiation, $D$, and convolution, $*$, are distribution operators as defined by L. Schwartz [1]. The guaranteed solution, $s$, will be a distribution with support in $J$, of order depending on the differentiability of $W$.

In recent treatments of the convolution equation over a compact interval by Shinbrot and Akutowicz [2], [3], [4], hypotheses are made on the Fourier transform of $W, \mathfrak{F} W$, which place restrictions on the real zeros of $\mathcal{F} W$ and exclude such kernels as $\mathcal{F}^{-1} \sin ^{2} x / x^{2}$. Now, the values of $W$ outside a neighborhood of $[-1,1]$ do not influence $W * s$ on $J$ if the distribution $s$ has support in $J$. The existence and nature of solutions of the convolution equation depends in principle, therefore, only upon $W$ as it is defined in a neighborhood of $[-1,1]$. Although in this paper we do not strictly generalize the results of [2], [3], [4], we do, in effect, restrict the values of $W$ only on a neighborhood of $[-1,1]$.

We employ Chover's idea [5], of assuming $W$ not differentiable at 0. $D^{2} W$ then has a point mass at 0 , and the operator $\left[D^{2} W *\right]$ can be written as the sum of two operators, one of which is the identity, $I$. In our theorems we assume that $D^{2} W=k-\delta_{0}-\psi$ on $[-1,1]$, where $k$ is a function of bounded variation and $\psi$ is a measure of variation less than 1. Results for kernels $W$ such that $D^{n} W$ has this form for some positive integer $n$ follow immediately as corollaries.

We are grateful to Professor J. Chover for his guidance and encouragement in this work. We also wish to thank Professor J. Korevaar for the present brief proof of Lemma 1.

2. Definitions. We use the term positive definite in the following sense. We call a continuous complex-valued function $W$ on the real

Received by the editors August 10, 1963.

1 Work on this paper was supported in part by a National Science Foundation Cooperative Graduate Fellowship and in part by Air Force ONR Contract No. AF49(638)-868. 
line, $R$, positive definite if for every nonzero Radon measure $m$ of compact support on $R, \iint W(x-y) d m(x) d \bar{m}(y)>0$. We use the equivalent form, $W * m * \tilde{m}(0)>0$, where $\tilde{m}$ denotes the reflection of $\bar{m}$ about 0 .

In expressions involving the distribution operators $D$ and $*$, we regard functions and measures as the associated distributions. We use several theorems found in [1] without specific reference. The primary one is that if $f$ and $g$ are distributions, at least one of which has compact support, then $D(f * g)=D f * g=f * D g$.

We -egard functions in $L^{2}(J, d x)$ as identically 0 outside of $J . \delta_{x}$ denotes the measure which is 0 except at $x$ where it has mass 1 . Hence $\left[\delta_{0} *\right]$ is the identity operator, $I$. By $\left[D^{2} W *\right]$ we mean the operator on $L^{2}(J, d x)$ which maps a function $f$ into the function which is $D^{2} W * f$ on $J$ and zero elsewhere. If $H \subset J, J-H=\{x: x \in J$ and $x \notin H\}$. If $T$ is an operator, $R(T)=$ range of $T$, and $N(T)=$ null space of $T$.

In Theorem 2 there appear distributions which are functions on the interior of $J$ and are extendable to functions of bounded variation on $J$. We call the class of such distributions B.V. $(J)$.

\section{Existence theorems and proofs.}

THEOREM 1. Let $W$ be a positive definite function on $[-1,1]$ such that $D^{2} W=k-\delta_{0}-\psi$, where $k$ is a function of bounded variation on $[-1,1]$ and $\psi$ is a measure on $[-1,1]$ of variation less than 1 . Then for any $g \in L^{2}(J)$ there exists $f \in L^{2}(J)$ such that $g=W * D^{2} f$ on $J$. If $g$ is a continuous function of bounded variation on $J$ then $f$ is also.

In the proof we use the following lemma.

Lemma 1. If $g=k * f-f-\psi * f$ on $J$, where $f \in L^{2}(J)$ and $g$ is a continuous function of bounded variation on $J$, then $f$ is a continuous function of bounded variation on $J$.

Proof. Since the convolution of two $L^{2}$ functions is continuous and $k$ has bounded variation, $k * f$ is a continuous function of bounded variation on $J$ and so is $k * f-g=f+\psi * f$. As is well known, $\operatorname{var} \psi<1$ implies that $\left[\psi_{*}\right]$ is an operator of norm $<1$ on $L^{2}(J)$ as well as on $C_{J}$. There exists $f_{1} \in C_{J}$ such that $f_{1}+\psi * f_{1}=f+\psi * f$ on $J$. Since $f_{1}$ and $f$ are both in $L^{2}(J), f_{1}=f$ and $f \in C_{J}$.

To show that $f$ is a function of bounded variation on $J$ it suffices to show that $\sum_{i=1}^{n}\left|f\left(x_{i}\right)-f\left(x_{i-1}\right)\right|$ is uniformly bounded for any $n$ and for any choice of $\left\{x_{i}, i=0, \cdots, n\right\} \subset J$. Let $v=\operatorname{var}_{J}(f+\psi * f)$. Fix $n$ and choose $\left\{x_{i}, i=0, \cdots, n\right\} \subset J$ such that 


$$
\sum_{i=1}^{n}\left|f\left(x_{i}\right)-f\left(x_{i-1}\right)\right|
$$

is maximized. Then

$$
\begin{aligned}
\sum_{i=1}^{n}\left|f\left(x_{i}\right)-f\left(x_{i-1}\right)\right| & \leqq v+\sum_{i=1}^{n} \int_{-1}^{1}\left|f\left(x_{i}-y\right)-f\left(x_{i-1}-y\right)\right| d \psi(y) \\
& \leqq v+\operatorname{var} \psi \cdot \sup _{y} \sum_{i=1}^{n}\left|f\left(x_{i}-y\right)-f\left(x_{i-1}-y\right)\right| .
\end{aligned}
$$

Since $g=0$ on the complement of $J$,

$$
\begin{aligned}
\sup _{y} \sum_{i=1}^{n}\left|f\left(x_{i}-y\right)-f\left(x_{i-1}-y\right)\right| \leqq & \max _{\left\{z_{i}\right\} \subset J} \sum_{i=1}^{n}\left|f\left(z_{i}\right)-f\left(z_{i-1}\right)\right| \\
& +\sup |f| .
\end{aligned}
$$

Therefore

$$
\sum_{i=1}^{n}\left|f\left(x_{i}\right)-f\left(x_{i-1}\right)\right| \leqq(v+\operatorname{var} \psi \cdot \sup |f|) /(1-\operatorname{var} \psi) .
$$

This completes the proof of the lemma.

To prove Theorem 1 we first note that since $W$ is positive definite, it is Hermitian, and $\left[D^{2} W *\right]=\left[\left(k-\delta_{0}-\psi\right) *\right]$ is a self-adjoint operator on $L^{2}(J)$. The closure of $R\left[D^{2} W *\right]$ is therefore the orthogonal complement of $N\left[D^{2} W *\right]$. We prove the first statement of the theorem by showing that $R\left[D^{2} W *\right]$ is closed and that $N\left[D^{2} W *\right]=0$. Then $R\left[D^{2} W *\right]=L^{2}(J)$ and for any $g \in L^{2}(J)$ there exists $f \in L^{2}(J)$ such that $g=D^{2} W * f=W * D^{2} f$.

If $T$ and $S$ are continuous linear operators on $L^{2}$ such that $T$ is completely continuous and $S^{-1}$ is continuous then $R[T-S]$ is closed. This can be shown by an argument closely analogous to that for the familiar case where $S$ is the identity. Since $k(x-y)$ is an element of $L^{2}([-1,1] \times[-1,1]),[k *]$ is a completely continuous operator on $L^{2}(J) .\left[\left(\delta_{0}+\psi\right) *\right]=I+[\psi *]$ has a continuous inverse. Therefore $R\left[[k *]-\left[\left(\delta_{0}+\psi\right) *\right]\right]$ is closed.

Suppose $f \in N\left[D^{2} W *\right]$, i.e., $k * f-f-\psi * f=0$ on $J$. By Lemma 1, $D f$ is a measure on $R$. Since $W$ is positive definite, $W * D f *(D f) \sim(0)$ $>0$ unless $D f \equiv 0 . \quad W * D f *(D f) \sim(0)=-\left(D^{2} W * f\right) * \tilde{f}(0)$ $=-\int_{-J}\left(D^{2} W * f\right)(-y) \bar{f}(-y) d y$ since the support of $\bar{f}$ is contained in $-J$. But since $D^{2} W * f=0$ on $J, W * D f *(D f)^{-}(0)=0$, and $D f \equiv 0$. $f$ has support in $J$. Therefore $D f \equiv 0$ implies $f \equiv 0$.

Suppose $g$ is a continuous function of bounded variation on $J$, and let $f$ be the element of $L^{2}(J)$ such that $g=D^{2} W * f=k * f-f-\psi * f$. 
By Lemma $1, f$ is a continuous function of bounded variation on $J$.

From this theorem we get, immediately:

Corollary 1. If $D^{n} W$ satisfies the hypothesis of Theorem 1, i.e., $D^{n} W$ is a positive definite function on $[-1,1]$ such that $D^{n+2} W=k-\delta_{0}$ $-\psi$ as stated, then for any $g \in L^{2}(J)$ there exists $f \in L^{2}(J)$ such that $g=W * D^{n+2} f$. If $g$ is a continuous function of bounded variation on $J$, then $D^{n+2} f$ is an $(n+1)$ st order distribution with support in $J$.

THEOREM 2. Let $H$ be a closed interval contained in the interior of $J$. Let $T$ be the self-adjoint operator on $L^{2}(J)$ defined by $T f=\left(k-\delta_{0}\right) * f$, where $k$ is a complex-valued, Hermitian function of bounded variation on $[-1,1]$. Then

(a) For any $g \in L^{2}(H)$ there exists $f \in L^{2}(J)$ such that $g=T f$ on $H$.

(b) If $g$ is a function of bounded variation on $H$, there exists $f \in L^{2}(J)$ of bounded variation such that $g=T f$ on $H$.

(c) If $D g$ is in B.V. $(H)$, there exists $f \in L^{2}(J)$ such that $D f$ is in B. V. $(J)$ and $g=T f$ on $H$.

In the proof of Theorem 2 we use the following lemma.

Lemma 2. If $g=k * f-f$ on $J$, where $D g$ is in B.V.(J) and $f$ is a measure with support in $J$ then $D f$ is in B.V.(J).

PROOF. Since $k * f$ and $g$ are both functions of bounded variation on $J, f$ is a function of bounded variation on $J$ and therefore on $R$. Hence, $D f$ is a measure, and $D(k * f)=k * D f$ is in B.V. $(J)$. Therefore so is $D f$, which proves the lemma.

To prove Theorem 2 we first note that $T$ is a self-adjoint operator on $L^{2}(J)$ and that $R(T)$ is closed and is the orthogonal complement of $N(T)$. Given $g \in L^{2}(H)$, we will construct an element of the orthogonal complement of $N(T)$ which equals $g$ on $H$.

Since $[k *]$ is a completely continuous operator on $L^{2}(J), N(T)$ is finite-dimensional. Choose $\left\{f_{i}, i=1, \cdots, n\right\}$, linearly independent on $J$ and spanning $N(T)$. Then the $f_{i}$ are linearly independent on $J-H$, as we now show.

Consider any $f=\sum_{i=1}^{n} c_{i} f_{i} . f \neq 0$ on $J$. Suppose $f=0$ on $J-H$. Then $f=0$ on $H^{c}=R-H . f$ is an element of $N(T)$, i.e., $k * f-f=0$ on $J$. Lemma 2, where $g=0$, implies that $D f$ is in B.V. $(J)$. Therefore $D^{2} f$ is a measure on the interior of $J . D^{2} f=0$ on $H^{c}$. Therefore $D^{2} f$ is a measure on $R$, with support in $J$. By Lemma 2, again, $D^{3} f$ is in B.V. $(J) . D^{4} f=0$ on $H^{c}$. Therefore $D^{4} f$ is a measure on $R$. Similarly, $D^{2 n f}$ is a measure on $R$ implies $D^{2 n+2} f$ is a measure on $R$, and so by induction $D^{n} f$ is a measure for all $n$. For any distribution $h, D h$ is a function implies $h$ is a continuous function, and $D h$ is a continuous 
function implies $h$ is differentiable and $D h=h^{(1)}$. Therefore $D^{n} f=f^{(n)}$, $f \in C^{\infty}$, and $f^{(n)} \in L^{2}(J)$ for all $n . f^{(n)}$ is, consequently, an element of the domain of $T$, and $T f^{(n)}=T D^{n} f=\left(k-\delta_{0}\right) * D^{n} f=D^{n}\left[\left(k-\delta_{0}\right) * f\right]$, which is 0 on the interior of $J$ because $\left(k-\delta_{0}\right) * f=0$ on $J$. Since $f$ is in $C^{\infty}$ on all of $R$, so is $D^{n}\left[\left(k-\delta_{0}\right) * f\right]$. Therefore $T f^{(n)}=0$ on $J$, i.e., $f^{(n)} \in N(T)$ for all $n$. But since $N(T)$ is finite-dimensional, there exists an integer $m$ and constants $b_{j}, j=0, \cdots, m$, not all zero, such that $\sum_{j=0}^{m} b_{j} f^{(j)}=0$ on $J$. Consequently $f$ is analytic on the interior of $J$ and cannot be $=0$ on $J-H$. It follows that the $f_{i}$ are linearly independent on $J-H$ as asserted.

The $f_{i}$ can be chosen so that their restrictions to $J-H$ form an orthonormal set. For instance, if $g_{i}=f_{i}-\sum_{j=1}^{i-1} a_{i j} g_{j}, i=1, \cdots, n$, is the Gram-Schmidt orthogonalization of the $f_{i}$ restricted to $J-H$, then the $g_{i}$ considered as functions on $J$ still span $N(T)$, since each $f_{i}$ is a linear combination of the $g_{i}$. We assume that the $f_{i}$ are so chosen.

Define

$$
G(x)=g(x) \text { for } x \in H, \quad G(x)=\sum_{i=1}^{n} b_{i} f_{i}(x) \text { for } x \in J-H,
$$

where $b_{i}=-\int_{H} f_{i}(x) g(x) d x$. Then $G \in L^{2}(J)$.

$$
\begin{aligned}
\int_{J} f_{i}(x) G(x) d x & =\int_{J-H} f_{i}(x)\left(\sum_{j=1}^{n} b_{j} f_{j}(x)\right) d x+\int_{H} f_{i}(x) g(x) d x \\
& =-b_{i}+b_{i}=0,
\end{aligned}
$$

for each $i=1, \cdots, n$. Therefore $G$ is in the orthogonal complement of $N(T)$ which as noted above is $R(T)$, i.e., there exists $f \in L^{2}(J)$ such that $G=T f$ on $J$. Since $g=G=T f$ on $H$, (a) is proved.

(b) Suppose $g$ is a function of bounded variation on $H$. If $f_{i} \in N(T)$, i.e., $k * f_{i}=f_{i}$ on $J$, then $f_{i}$ is a function of bounded variation. Therefore $G$, as defined above, is a function of bounded variation, and $T f=k * f-f=G$ on $J$ implies $f$ is a function of bounded variation on $J$ and therefore on $R$.

(c) Suppose $D g$ is in B.V.(H). If $f_{i} \in N(T)$ then Lemma 2 implies that $D f_{i}$ is in B.V. $(J)$. Therefore $G$ as defined above is such that $D G$ can fail to be in B.V. $(J)$ only if $G$ has discontinuities at the endpoints, $c$ and $d$, of $H$. To remedy this we will alter the definition of $G$ on $J-H$. Let $h(x)$ be a function on $J-H$ such that

(i) $h$ is in the orthogonal complement of $N(T)$,

(ii) $D h$ is a function of bounded variation on $J-H$,

(iii) $h(c)=g(c)-\sum_{i=1}^{n} b_{i} f_{i}(c), h(d)=g(d)-\sum_{i=1}^{n} b_{i} f_{i}(d)$.

\section{Define}


$G(x)=\sum_{i=1}^{n} b_{i} f_{i}(x)+h(x)$ for $x \in J-H, \quad G(x)=g(x)$ for $x \in H$.

Then $D G$ is in B.V. $(J)$, and $G$ is in the orthogonal complement of $N(T)$. There exists $f \in L^{2}(J)$ such that $G=k * f-f$ on $J$. Lemma 2 implies that $D f$ is in B.V. $(J)$.

We obtain immediately from Theorem 2 .

Corollary 2. If $W$ is a function on $[-1,1]$ such that $D^{n} W=k-\delta_{0}$ for some positive integer $n$, where $k$ is as in Theorem 2 , then for every $g \in L^{2}(H)$ there exists $f \in L^{2}(J)$ such that $g=W * D^{n} f$ on $H$.

\section{REFERENCES}

1. L. Schwartz, Theorie des distributions, Vol. I, Actualités Sci. Indust. No. 1091, Hermann, Paris, 1950; Vol. II, Actualites Sci. Indust. No. 1122, Hermann, Paris, 1951.

2. M. Shinbrot, Difference kernels, Stanford Univ. Appl. Math. and Statist. Laboratories Tech. Rep. No. 92, Stanford Univ., Stanford, Calif., 1960. 406.

3. —_, A classing of difference kernels, Proc. Amer. Math. Soc. 13 (1962), 399-

4. E. J. Akutowicz, On approximation with respect to certain quadratic norms, J. Math. Mech. 11 (1962), 153-164.

5. J. Chover, $A$ theorem on integral transforms with an application to prediction theory, J. Math. Mech. 8 (1959), 939-946.

UNIVERSITY OF WISCONSIN 